Received: 04.11.2019

Revised: 04.12.2019

Accepted: 20.12 .2019

DOI: $10.17804 / 2410-9908.2019 .6 .037-047$

\title{
SPECIFIC FEATURES OF GRAIN STRUCTURE EVOLUTION IN HPT-NANOSTRUCTURED TIN BRONZE UNDER SUBSEQUENT HEATING
}

\author{
A. V. Stolbovsky ${ }^{\text {a)* }}$, V. V. Popov ${ }^{\text {b) }}$, R. M. Falakhutdinov ${ }^{\text {c) }}$, and S. A. Murzinova ${ }^{\text {d) }}$ \\ M. N. Miheev Institute of Metal Physics, Ural Branch of the Russian Academy of Sciences, \\ 18 S. Kovalevskoy St., Ekaterinburg, 620108, Russian Federation \\ a) iD http://orcid.org/0000-0001-5228-7857 stolbovsky@imp.uran.ru; \\ b) iD http://orcid.org/0000-0002-9291-0102 vpopov@imp.uran.ru; \\ c) iD http://orcid.org/0000-0001-8505-9455 falahutdinov@imp.uran.ru; \\ d) (iD http://orcid.org/0000-0001-5240-2966 murzinova@imp.uran.ru \\ *Corresponding author. E-mail: stolbovsky@imp.uran.ru
}

Address for correspondence: ul. S. Kovalevskoy, 18, Ekaterinburg, 620108, Russian Federation Tel.: +7 34337835 74; fax: +7 3433745244

The grain structure of tin bronze with $7.4 \mathrm{wt} \% \mathrm{Sn}$ after high-pressure torsion (HPT) at room temperature and subsequent annealing is analyzed. It is demonstrated that, in $\mathrm{Cu}-7.4 \% \mathrm{Sn}$ bronze, two groups of grains with different characteristics and different grain-boundary mobility are formed under deformation by HPT. It can be stated that the formation of two groups of grains results from different inclination of grains to relaxation due to the presence of competitive processes occurring directly under deformation. The grains of both groups evolve under heating, with increasing average crystallite size as the annealing temperature rises; however, their volume fraction depends on the defectiveness of the crystallites themselves.

Keywords: nanostructuring, nanostructures, severe plastic deformation, high-pressure torsion, grain boundaries, thermal stability, tin bronze, statistical analysis.

\section{Acknowledgment}

The electron microscope investigation was performed on the equipment installed in Nanotechnologies and Advanced Materials Testing Center, IMP UB RAS.

The study was performed under the state assignment of from FASO Russia (theme Function, No. AAAA-A19-119012990095-0) and partially supported by the Basic Research Program of UB RAS, project 18-10-2-37.

\section{References}

1. Gleiter H. Nanostructured materials: basic concepts and microstructure. Acta Mater., 2000, vol. 48, no. 1, pp. 1-29. DOI: 10.1016/S1359-6454(99)00285-2.

2. $\quad$ Valiev R.Z., Zhilyaev A.P., Langdon T.G. Bulk nanostructured materials: Fundamentals and applications, Hoboken, New Jersey, USA, TMS, Wiley, 2013, pp. 440. DOI: 10.1002/9781118742679.

3. Estrin Y., Vinogradov A. Extreme grain refinement by severe plastic deformation: A wealth of challenging science. Acta Materialia, 2013, vol. 61, iss. 3, pp. 782-817. DOI: $10.1016 /$ j.actamat.2012.10.038.

4. Sauvage X., Wilde G., Divinski S.V., Horita Z., Valiev R.Z. Grain boundaries in ultrafine grained materials processed by severe plastic deformation and related phenomena. Mater. Sci. Eng. A., 2012, vol. 540, pp. 1- 12. DOI: 10.1016/j.msea.2012.01.080. 
5. Popov V.V., Sergeev A.V., Stolbovsky A.V. Emission Mцssbauer spectroscopy of grain boundaries in ultrafine-grained $\mathrm{W}$ and Mo produced by severe plastic deformation. Physics of Metals and Metallography, 2017, vol. 118, pp. 354-361. DOI: https://doi.org/10.1134/S0031918X17040081.

6. Stolbovskii A.V., Popova E.N. Study of the Grain Boundary Structure in Submicrocrystalline Niobium after Equal-Channel Angular Pressing. Bulletin of the Russian Academy of Sciences: Physics, 2010, vol. 74, iss. 3, pp. 388-392. DOI: 10.3103/S1062873810030159.

7. Popov V.V., Sergeev A.V., Stolbovsky A.V. Emission Nuclear Gamma-Resonance Spectroscopy of Grain Boundaries in Coarse-Grained and Ultrafine-Grained Polycrystalline Mo. Defect and Diffusion Forum, 2015, vol. 364, pp. 147-156. DOI: 10.4028/www.scientific.net/DDF.364.147. 8. Popov V.V., Stolbovsky A.V., Sergeev A.V., Semionkin V.A. Mцssbauer Spectroscopy of Grain Boundaries in Ultrafine-Grained Materials Produced by Severe Plastic Deformation. Bulletin of the Russian Academy of Sciences: Physics, 2017, vol. 81, iss. 7, pp. 951-955. DOI: $10.3103 / \mathrm{S} 106287381707022 \mathrm{X}$.

9. Popov V.V., Stolbovsky A.V., Popova E.N., Pilyugin V.P. Structure and thermal stability of $\mathrm{Cu}$ after severe plastic deformation. Defect and Diffusion Forum, 2010, vols. 297-301, pp. 1312-1321. DOI: 10.4028/www.scientific.net/DDF.

10. Stolbovsky A.V., Popov V.V., Popova E.N., Pilyugin V.P. Structure, thermal stability, and state of grain boundaries of copper subjected to high-pressure torsion at cryogenic temperatures. Bulletin of the Russian Academy of Sciences: Physics, 2014, vol. 78, iss. 9, pp. 908-916. DOI: $10.3103 / \mathrm{S} 1062873814090299$.

11. Pippan R., Scheriau S., Taylor A., Hafok M., Hohenwarter A., Bachmaier A. Saturation of fragmentation during severe plastic deformation. Annual Review of Materials Research, 2010, vol. 40, pp. 319-343. DOI: 10.1146/annurev-matsci-070909-104445.

12. Stolbovsky A.V., Popov V.V., Popova E.N. Structure and Thermal Stability of Tin Bronze Nanostructured by High Pressure Torsion. Diagnostics, Resource and Mechanics of materials and structures, 2015, iss. 5, pp. 118-132. DOI: 10.17804/2410-9908.2015.5.118-132. URL: http://dream-journal.org/issues/2015-5/2015-5_52.html (accessed 30.10.2017).

13. Popov V.V., Popova E.N., Stolbovsky A.V., Falahutdinov R.M. Evolution of the Structure of $\mathrm{Cu}-1 \%$ Sn Bronze under High Pressure Torsion and Subsequent Annealing. Physics of Metals and Metallography, 2018, vol. 119, pp. 358-367. DOI: 10.1134/S0031918X18040154.

14. Popov V.V., Stolbovsky A.V., Popova E.N. Structure of nickel-copper alloys subjected to high-pressure torsion to saturation stage. Physics of Metals and Metallography, 2017, vol. 118, pp. 1073-1080. DOI: https://doi.org/10.1134/S0031918X17110114.

15. Popov V.V., Stolbovsky A.V., Popova E.N., Pilyugin V.P. Structure and thermal stability of $\mathrm{Cu}$ after severe plastic deformation. Defect and Diffusion Forum, 2010, vol. 297-301, pp. 1312-1321. DOI: 10.4028/www.scientific.net/DDF.

16. Stolbovsky A.V., Popov V.V., Popova E.N., Pilyugin V.P. Structure, thermal stability, and state of grain boundaries of copper subjected to high-pressure torsion at cryogenic temperatures. Bulletin of the Russian Academy of Sciences: Physics, 2014, vol. 78, iss. 9, pp. 908-916. DOI: $10.3103 / \mathrm{S} 1062873814090299$.

17. Kon'kova T.N., Mironov S.Y., Korznikov A.V. Room-temperature instability of the structure of copper deformed at a cryogenic temperature. Russian Metallurgy (Metally), 2011, vol. 2011, iss. 7, pp. 689-698. DOI: 10.1134/S0036029511070081.

18. Voronova L.M., Chashchukhina T.I., Degtyarev M.V., Pilyugin V.P. Structure Evolution and Stability of Copper Deformed at 80 K. Russian Metallurgy (Metally), 2012, vol. 2012, iss. 4, pp. 303-306. DOI: 10.1134/S0036029512040131.

19. Chashchukhina T.I., Voronova L.M., Degtyarev M.V., Pokryshkina D.K. Deformation and dynamic recrystallization in copper at different deformation rates in Bridgman anvils, Physics of Metals and Metallography, 2011, vol. 111, iss. 3, pp. 304-313. DOI: 10.1134/S0031918X11020049. 
20. Stolbovsky A., Farafontova E. Statistical analysis method of the grain structure of nanostructured single phase metal materials processed by high-pressure torsion. Sol. Stat. Phenomena, 2018, vol. 284, pp. 425-430. DOI: https://doi.org/10.4028/www.scientific.net/SSP.284.425.

21. Stolbovsky A., Farafontova E. Statistical analysis of histograms of grain size distribution in nanostructured materials processed by severe plastic deformation. Sol. Stat. Phenomena, 2018, vol. 284, pp. 431-435. DOI: https://doi.org/10.4028/www.scientific.net/SSP.284.431. 
Подана в журнал: 04.11.2019

УДК 669.3'6:539.89:539.25

DOI: $10.17804 / 2410-9908.2019 .6 .037-047$

\title{
ОСОБЕННОСТИ ЭВОЛЮЦИИ ЗЕРЕННОЙ СТРУКТУРЫ НАНОСТРУКТУРИРОВАННОЙ МЕТОДОМ КВД ОЛОВЯНИСТОЙ БРОНЗЫ ПРИ ПОСЛЕДУЮЩЕМ НАГРЕВЕ
}

\author{
А. В. Столбовский ${ }^{\mathrm{a}}{ }^{*}$, В. В. Попов ${ }^{\text {б) }}$, Р. М. Фалахутдинов ${ }^{\text {в) }}$, С. А. Мурзинова ${ }^{\text {г) }}$ \\ Институт физики металлов им. М.Н. Михеева УрО РАН, \\ д. 18, ул. С. Ковалевской, Россия, 620108, г. Екатеринбург, Российская Федерация

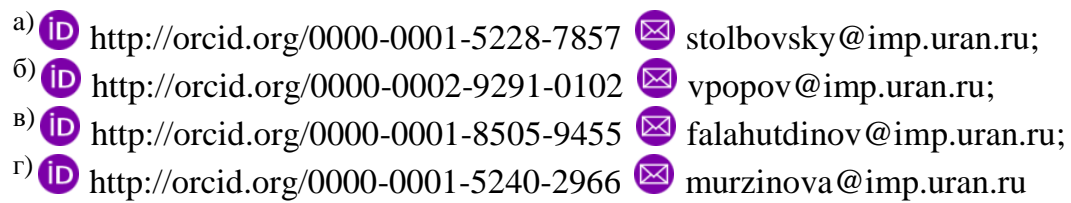 \\ *Ответственный автор. Электронная почта: stolbovsky@imp.uran.ru \\ Адрес для переписки: ул. С. Ковалевской, 18, Екатеринбург, 620108, Российская Федерация \\ Тел.: +7 (343) 378-35-74; факс: (343) 374-52-44
}

Проведен анализ зеренной структуры наноструктурированной оловянистой бронзы с 7,4 мас. \% Sn после деформации кручением под высоким давлением (КВД) при комнатной температуре и последующего отжига. Показано, что в оловянистой бронзе $\mathrm{Cu}-7,4$ \% Sn при деформации формируются две группы зерен с различными характеристиками, обладающие различной подвижностью границ зерен. Можно констатировать, что образование двух групп зерен является следствием присутствия конкурирующих процессов, протекающих непосредственно при деформации, что и определяет их различную склонность к релаксации при последующем нагреве. При этом зерна обеих выделенных групп эволюционируют при нагреве с возрастанием среднего размера кристаллитов с повышением температуры отжига, однако их объемная доля зависит от дефектности самих кристаллитов.

Ключевые слова: наноструктурирование, наноструктуры, интенсивная пластическая деформация, кручение под высоким давлением, границы зерен, термическая стабильность, оловянистая бронза, статистический анализ.

\section{1. Введение}

Применение интенсивной пластической деформации (ИПД) для наноструктурирования материалов позволяет получать материалы с необычным механическим поведением, уникальной структурой и свойствами [1-3]. Показано, что границы зерен в материалах, полученных ИПД, значительно отличаются от границ зерен в обычных поликристаллах [4-8]. Считается, что границы зерен деформационного происхождения в нано- и субмикрокристаллических металлических материалах характеризуются высокой плотностью дефектов кристаллического строения и, соответственно, высокой энергией.

В то же время одной из важнейших проблем в создании наноструктурированных материалов с особыми свойствами является низкая термическая стабильность получаемых структур, особенно в чистых металлах [9-11]. Переход к материалам с увеличенной концентрацией примесных атомов в меди выражается в очевидном улучшении ее способности к наноструктурированию и к получению наноструктурного состояния уже при относительно малой степени деформации [12-14]. Это позволяет проследить эволюцию деформированной структуры именно при нагреве, поскольку полученные структуры сохраняются при комнатной температуре в отличие от наноструктурированной меди [15-19]. 
Протекание конкурирующих процессов во время интенсивной пластической деформации материалов, когда вместе с формированием границ деформационного происхождения идет конкурирующий процесс их релаксации [11], обусловливает образование как зерен, образованных границами, сформированными деформационным путем, так и зерен, сформированных в результате релаксационных процессов. Более того, различный механизм формирования таких зерен подразумевает и различное состояние границ, формирующих данные кристаллиты [2-8].

Использование ядерной гамма-резонансной спектроскопии позволяет различать границы зерен с различного происхождения по состоянию находящихся в границе зерна и приграничных объемах меченных атомов, которое зависит от их окружения [5-8], однако такой метод является уникальным и не имеет широкого применения из-за его высокой сложности. Поэтому в работах, посвященных структурным исследованиям наноструктурированных материалов, наиболее часто используется просвечивающая электронная микроскопия (ПЭМ).

Использование методики последующей обработки снимков, полученных с помощью ПЭМ, с построением распределений кристаллитов по их размерам дает возможность судить о среднем размере зерен структуры и изменении ее параметров при дальнейшем нагреве. Однако такой подход не позволяет произвести детальный анализ с возможностью разделения, если это необходимо, зерен на группы по их особенностям, в том числе по природе происхождения. Такое разделение является крайне интересным, поскольку процессы, проходящие в материале при деформации, например методом КВД, являются во многом конкурирующими, с проявлением различных релаксационных процессов, в том числе происходящих непосредственно после деформации или при последующем отжиге [11-19].

Медь и однофазные сплавы на основе меди являются интересным модельным материалом. Для исследования был выбран состав, близкий к границе максимальной растворимости олова в меди, имеющий однофазную структуру в широком диапазоне температур. Это позволяет получать, в отличие от чистой меди, устойчивое наноструктурное состояние и проследить эволюцию структуры при дальнейшем отжиге [12].

В работе [20] был предложен метод характеризации зеренной структуры, основанный на статистическом анализе распределений зерен по размерам для материалов, полученных ИПД. Однако статистический анализ был проведен только с использованием нормального распределения, в то время как в работе [21] была предложена модель, позволяющая повысить точность подгонки. В настоящей работе были поставилены задачи объединить разработанные подходы в единую методику и детально проанализировать эволюцию структуры при нагреве оловянистой бронзы, наноструктурированной методом КВД.

\section{2. Материал и методика исследования}

Для исследований использовались бронза с содержанием 7,4 мас. \% олова. Сплав $\mathrm{Cu}$ 7,4 \% Sn в литом состоянии подвергали гомогенизирующему отжигу в вакуумной печи при температуре $750{ }^{\circ} \mathrm{C}$ в течение 2 ч, чтобы исключить влияние дендритной ликвации и выровнять состав по сечению образцов. Для деформации кручением под высоким давлением вырезали диски диаметром 10 мм и толщиной 0,5 мм. Полученные диски деформировали в наковальнях Бриджмена под давлением 4 ГПа с угловой скоростью 0,3 об./мин на 5 об. при комнатной температуре.

Структуру деформированных и отожженных образцов изучали с помощью просвечивающей электронной микроскопии в электронных микроскопах Philips-CM30 SuperTwin и JEM-200CX с дальнейшей обработкой снимков в автоматизированном комплексе SIAMS-600 для построения распределения зерен по размерам. 


\section{3. Результаты исследования и их обсуждение}

В обзоре [11] было предложено трактовать получаемые при ИПД кручением структуры как результат воздействия конкурирующих процессов во время деформирования материала, когда вместе с формированием границ деформационного происхождения идет конкурирующий процесс их релаксации. Таким образом, в структуре предположительно должны наблюдаться как зерна, образованные границами, сформированными деформационным путем, так и зерна, сформированные в результате релаксационных процессов. Более того, различный механизм формирования таких зерен подразумевает и различное состояние границ, формирующих данные кристаллиты. Такой подход позволяет сделать акцент именно на неравновесности формирующихся границ, состояние которых значительно отличается от границ в крупнозернистых поликристаллах, формирующихся вследствие рекристаллизации [5-8].

В то же время в материалах, полученных методом КВД, обладающих средним размером кристаллитов около и менее 100 нм, визуальное разделение невозможно, это можно увидеть в работах по широкому спектру чистых металлов и однофазных сплавов [12-19], где представлены распределения зерен по размерам. При этом в большинстве этих работ высказывается предположение об однородности получаемой зеренной структуры. В работах $[18,19]$ использовалась аппроксимация полученных гистограмм единственным распределением. И в основном анализ распределения сводился к утверждению, что получаемые при КВД распределения зерен по размерам аппроксимируются логарифмически нормальным распределением.

В работе предлагается разделять зерна на отдельные группы кристаллитов по склонности их к релаксационным процессам при дальнейшем нагреве в рамках модельного подхода [20].

На рис. 1 приведены исходные гистограммы распределения зерен по размерам с наложением на гистограмму кривых, отвечающих отдельным распределениям и их суммарной кривой, а также представлена анализируемая структура.

Группы кристаллитов аппроксимировались моделью, представленной в работе [21], следующего вида:

$$
f(x)=A \cdot \frac{1}{x} \cdot \frac{1}{\sqrt{2 \pi} \cdot \sigma} \cdot e^{-\frac{(\ln x-\mu)^{2}}{2 \sigma^{2}}}+B \cdot \frac{1}{\sqrt{2 \pi} \cdot S} \cdot e^{-\frac{(x-M)^{2}}{2 S^{2}}},
$$

где $M=e^{\mu+\frac{\sigma^{2}}{2}}$ - среднее значение для компоненты модели нормального распределения, рассчитанное из соответствующей величины для логарифмического нормального распределения; $S=\sqrt{\left(e^{\sigma^{2}}-1\right) \cdot e^{2 \cdot \mu+\sigma^{2}}}-$ стандартное отклонение $(\mathrm{CКО})$, также рассчитанное из соответствующей величины для логарифмического нормального распределения; $\mu, \sigma$ - соответственно, среднее значение и стандартное отклонение для логнормального распределения; $A$ и $B$ - размерные факторы, определяющие вклад соответствующей компоненты.

Этот подход позволяет наиболее точно описать именно отдельную группу зерен, поскольку как логнормальное, так и нормальное распределения по отдельности не могут точно описать экспериментальное распределение, как было показано и подробно описано в работе [21], поэтому был сделан выбор в пользу более сложного, но при этом более точного модельного описания. 

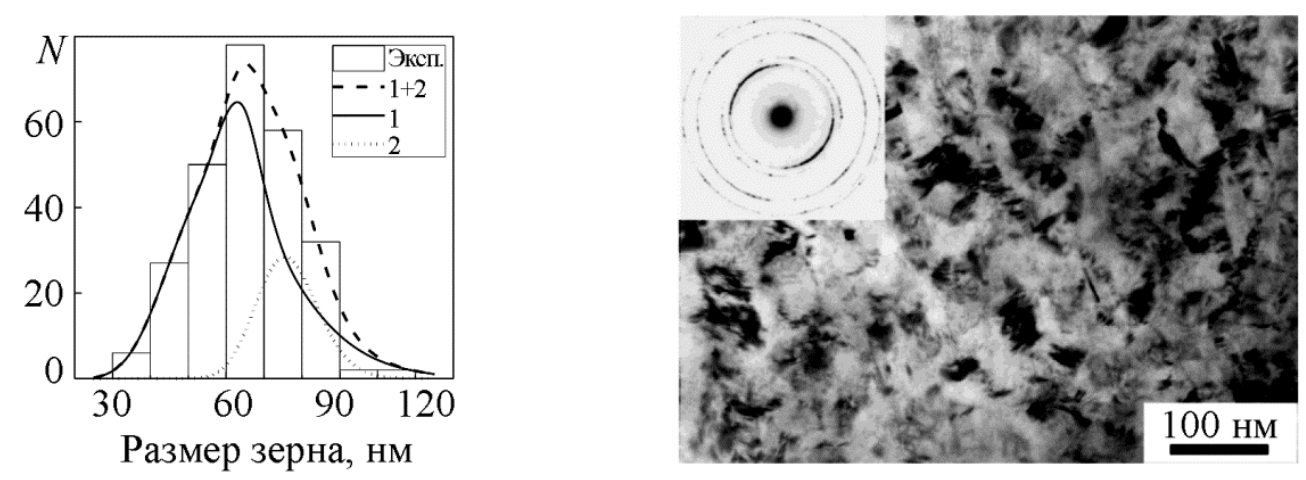

$a$
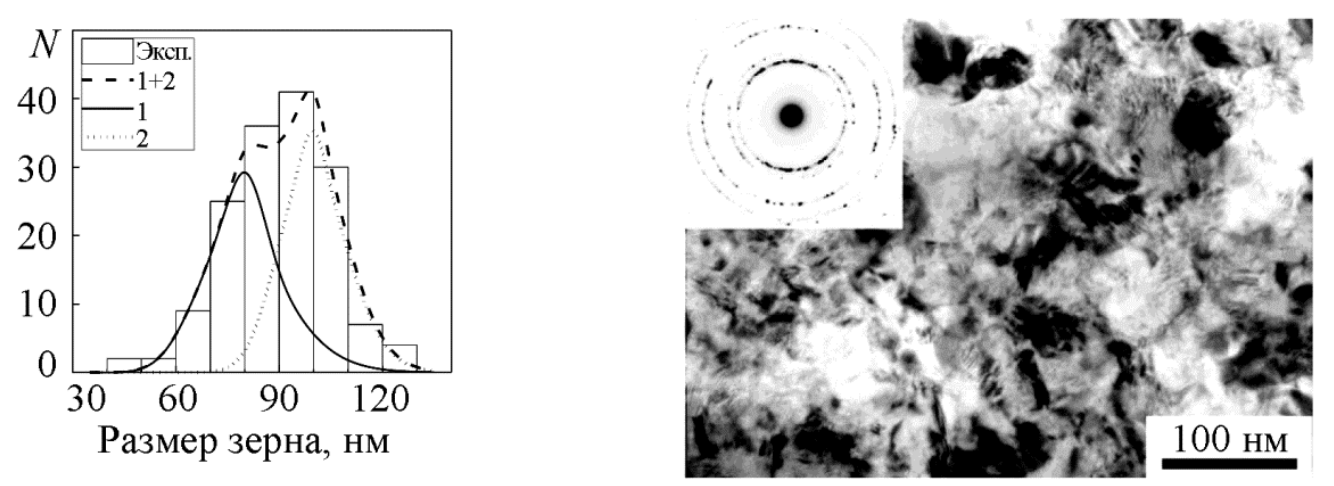

$\sigma$
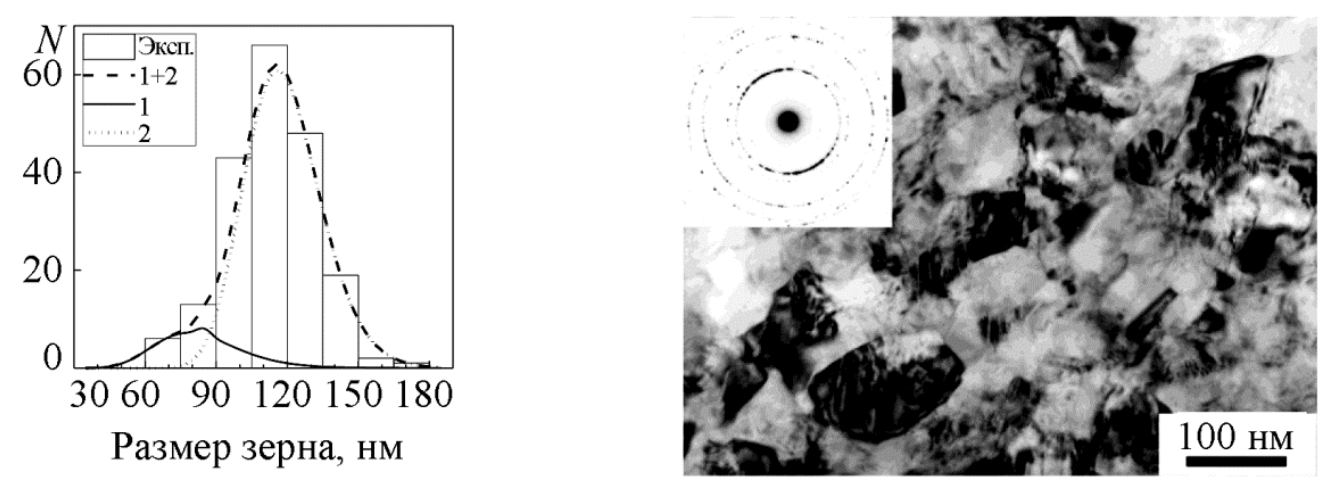

B
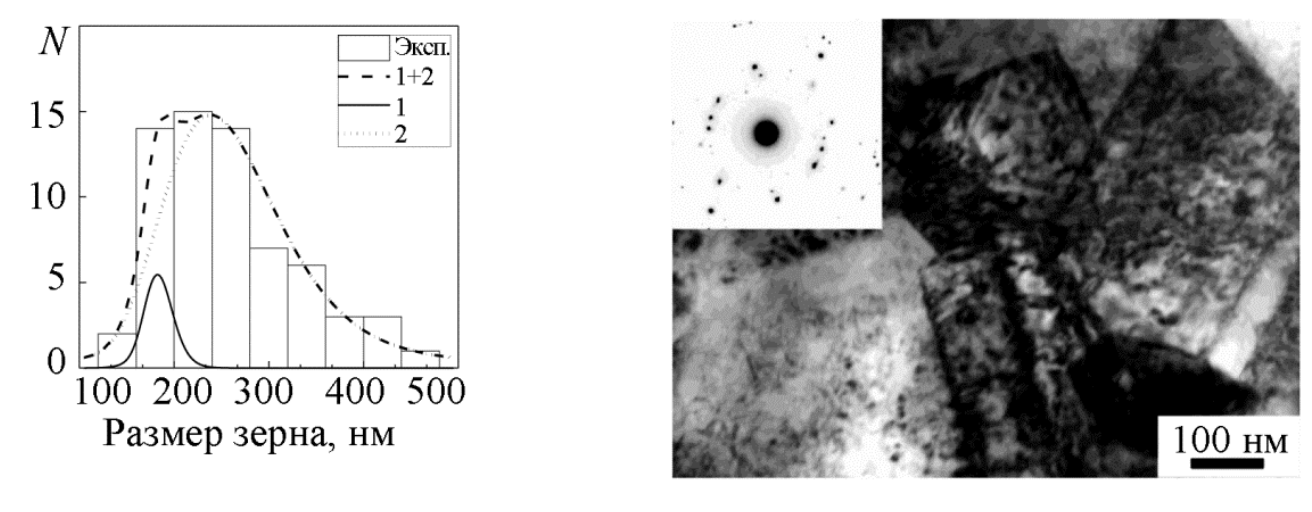

2

Рис. 1. Гистограммы распределения зерен по размерам с выделением отдельных групп зерен и микроструктура образцов при различной обработке: $a-\mathrm{KBД;} \sigma-200{ }^{\circ} \mathrm{C}$;

$$
\text { в }-250{ }^{\circ} \mathrm{C} ; 2-300{ }^{\circ} \mathrm{C}
$$


Результаты расчетов представлены на рис. 2. Помимо зависимостей среднего размера зерна, СКО и объемной доли отдельных распределений показаны также доли нормального и логнормального распределений для каждой отдельно выделенной группы зерен, в соответствии с моделью, представленной в работе [21]. Это позволяет провести анализ по дополнительным параметрам, относящимся к особенностям каждого отдельного распределения и соответственно связанным с природой формирования данных групп зерен.
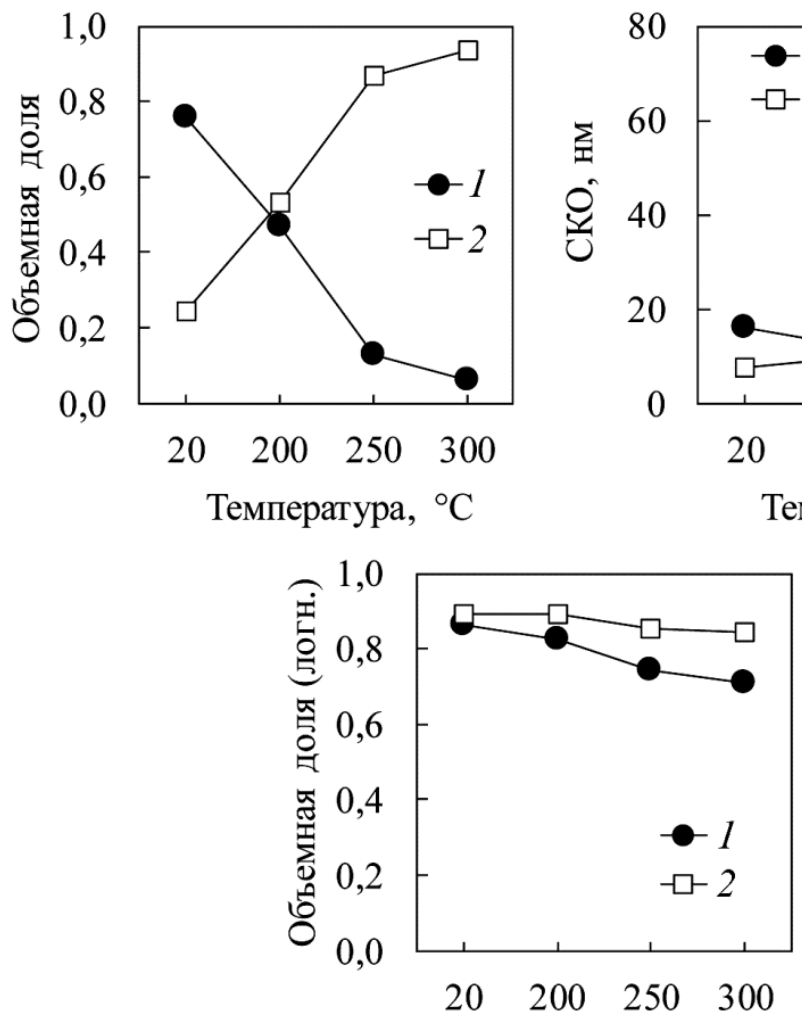

Температура, ${ }^{\circ} \mathrm{C}$
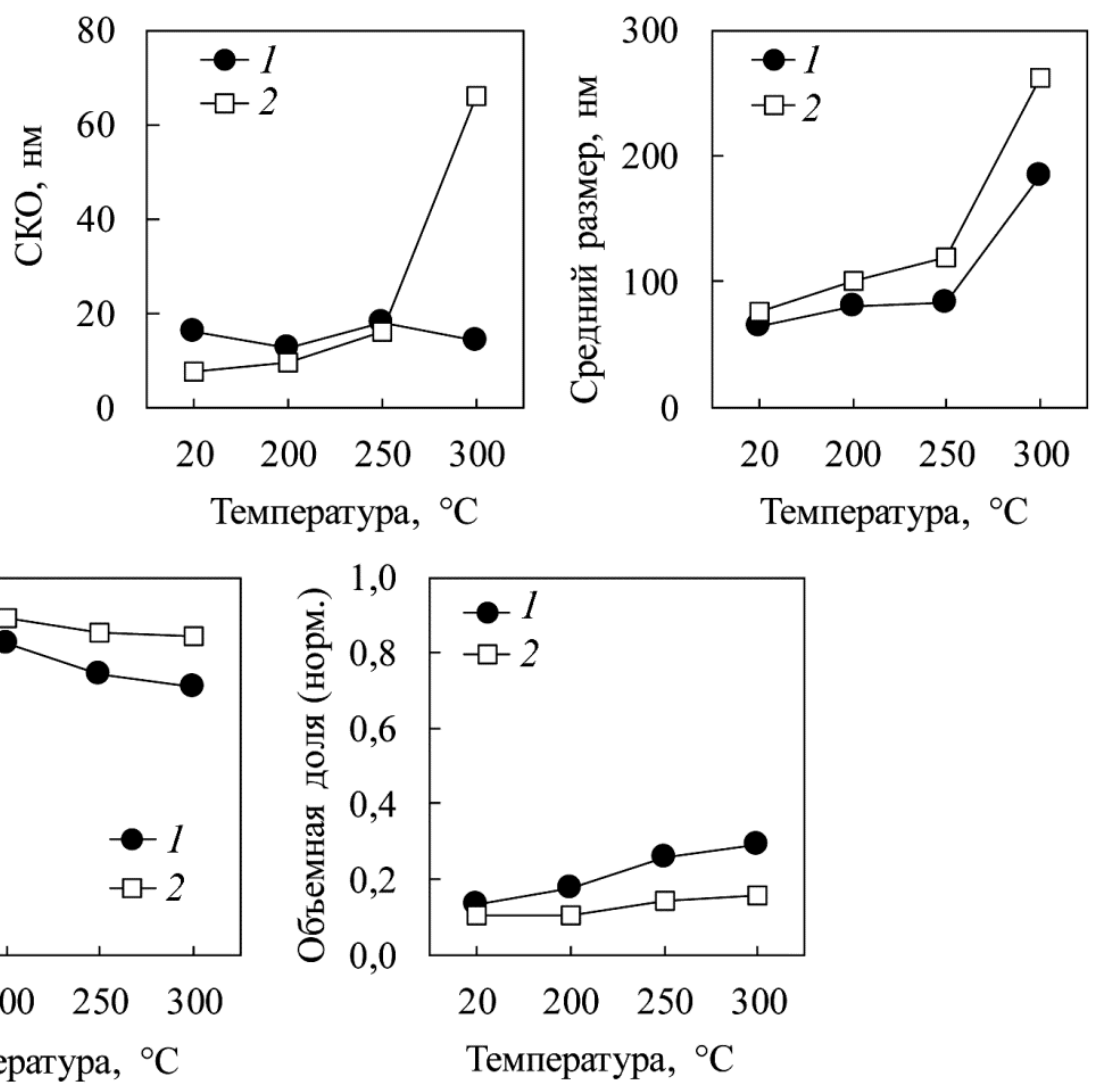

Рис. 2. Зависимости расчетных параметров распределений от температуры термической обработки $(1,2$ - группы зерен)

По изменению значения объемной доли и среднего размера зерна можно выделить две отдельные группы зерен. С повышением температуры отжига средний размер зерна постепенно увеличивается для обеих групп зерен до температуры отжига $250{ }^{\circ} \mathrm{C}$. Структуры имеют схожий характер с постепенно увеличивающимся размером кристаллитов. В то время как начиная с $300{ }^{\circ} \mathrm{C}$ происходит резкое увеличение среднего размера кристаллитов. Если проследить изменение объемных долей обеих групп, можно увидеть, что объемная доля одной из групп постепенно снижается без резких изменений при всех температурах отжига. Соответственно, объемная доля другой группы кристаллитов постепенно увеличивается.

Необходимо отметить, что модель включает дополнительные статистические характеристики, такие как доли логнормального и нормального распределений для каждой группы зерен. Это позволяет делать заключение об отнесении выделенного набора кристаллитов к группе 1 или 2, основываясь на очень близких параметрах, характерных для соответствующей группы. На рис. 2 видно, что доли логнормального и нормального распределений для каждой из выделенных групп зерен при данных температурах отжига имеют очень близкие параметры. Такое постоянство долей компонентов распределений может также указывать на идентичную природу происхождения выделенных групп зерен. 
Таким образом, по результатам анализа можно утверждать, что сформированные группы зерен эволюционируют при всех исследованных температурах. Сильное различие в изменении СКО для выделенных групп кристаллитов при температуре $300{ }^{\circ} \mathrm{C}$ при значительном росте среднего значения можно объяснить сложностью подгонки вследствие малой объемной доли группы 1 . В то же время значительное повышение СКО для группы 2 укладывается в тренд и связано с изменением среднего размера данной группы кристаллитов.

Значительное возрастание объемной доли и последующее абсолютное доминирование группы 2 при нагреве дает все основания утверждать, что кристаллиты, формирующие данную группу, более склонны к протеканию релаксационных процессов, обладают меньшей дефектностью внутреннего строения и сформированы в границах с меньшей энергией, что делает их предпочтительнее для системы с энергетической точки зрения. В то же время группу 1 образуют кристаллиты с большей дефектностью внутреннего строения и большей энергией границ зерен, что приводит к уменьшению их объемной доли при последующем нагреве. При этом кристаллиты также эволюционируют при нагреве, поскольку их средний размер возрастает с повышением температуры отжига, однако их объемная доля снижается и при дальнейшем повышении температуры отжига данная группа кристаллитов либо должна полностью исчезнуть, либо быть неотличима от доминирующей группы.

\section{4. Заключение}

Применение комплексного статистического подхода с подробным описанием гистограмм распределения зерен по размерам позволяет проводить анализ зеренной структуры в однофазных металлических материалах, наноструктурированных методом КВД.

Использование дополнительных статистических характеристик, таких как доли логнормального и нормального распределений для каждой группы зерен, можно использовать как дополнительные особенности для разделения экспериментально определенного распределения на группы при эволюции структуры.

Показано, что в оловянистой бронзе состава $\mathrm{Cu}-7,4$ \% $\mathrm{Sn}$ при деформации формируются две группы зерен с различными характеристиками, обладающие различной подвижностью границ зерен. При этом можно утверждать, что формирование двух групп обусловлено именно различной склонностью зерен к протеканию релаксационных процессов как следствие присутствия конкурирующих процессов, проходящих непосредственно во время деформирования.

В то же время зерна обеих выделенных групп эволюционируют при нагреве с возрастанием среднего размера кристаллитов с повышением температуры отжига, однако их объемная доля зависит от дефектности самих кристаллитов. Чем ниже дефектность кристаллитов, тем больше будет их объемная доля при последующем нагреве.

\section{Благодарность}

Электронно-микроскопическое исследование выполнено на оборудовании центра коллективного пользования в Испытательном иентре нанотехнологий и перспективных материалов ИФМ УрО РАН.

Работа выполнена в рамках государственного задания ФАНО России (тема «Функичя» номер госрегистрации ААAA-A19-119012990095-0), при частичной поддержке программы фундаментальных исследований УрО РАН (проект 18-10-2-37).

\section{Литература}

1. Литература Gleiter H. Nanostructured materials: basic concepts and microstructure // Acta Mater. - 2000. - Vol. 48, no. 1. - P. 1-29. - DOI: 10.1016/S1359-6454(99)00285-2. 
2. Valiev R. Z., Zhilyaev A. P., Langdon T. G. Bulk nanostructured materials: Fundamentals and applications. - Hoboken, New Jersey, USA : TMS, Wiley, 2013. - P. 440. DOI: $10.1002 / 9781118742679$.

3. Estrin Y., Vinogradov A. Extreme grain refinement by severe plastic deformation: A wealth of challenging science. - Acta Materialia. - 2013. - Vol. 61, iss. 3. - P. 782-817. DOI: 10.1016/j.actamat.2012.10.038.

4. Grain boundaries in ultrafine grained materials processed by severe plastic deformation and related phenomena / X. Sauvage, G. Wilde, S. V. Divinski, Z. Horita, R. Z. Valiev // Mater. Sci. Eng. A. - 2012. - Vol. 540. - P. 1- 12. - DOI: 10.1016/j.msea.2012.01.080.

5. Popov V. V., Sergeev A. V., Stolbovsky A. V. Emission Mцssbauer spectroscopy of grain boundaries in ultrafine-grained $\mathrm{W}$ and Mo produced by severe plastic deformation // Physics of Metals and Metallography. - 2017. - Vol. 118. - P. 354-361. - DOI: https://doi.org/10.1134/S0031918X17040081.

6. Stolbovskii A. V., Popova E. N. Study of the Grain Boundary Structure in Submicrocrystalline Niobium after Equal-Channel Angular Pressing // Bulletin of the Russian Academy of Sciences: Physics. - 2010. - Vol. 74, iss. 3. - P. 388-392. - DOI: 10.3103/S1062873810030159.

7. Popov V. V., Sergeev A. V., Stolbovsky A. V. Emission Nuclear Gamma-Resonance Spectroscopy of Grain Boundaries in Coarse-Grained and Ultrafine-Grained Polycrystalline Mo // Defect and Diffusion Forum. - 2015. - Vol. 364. - P. 147-156. - DOI: 10.4028/www.scientific.net/DDF.364.147.

8. Mцssbauer Spectroscopy of Grain Boundaries in Ultrafine-Grained Materials Produced by Severe Plastic Deformation / V. V. Popov, A. V. Stolbovsky, A. V. Sergeev, V. A. Semionkin // Bulletin of the Russian Academy of Sciences: Physics. - 2017. - Vol. 81, iss. 7. - P 951-955. DOI: $10.3103 / \mathrm{S} 106287381707022 \mathrm{X}$.

9. Structure and thermal stability of $\mathrm{Cu}$ after severe plastic deformation / V. V. Popov, A. V. Stolbovsky, E. N. Popova, V. P. Pilyugin // Defect and Diffusion Forum. - 2010. Vols. 297-301. - P. 1312-1321. - DOI:10.4028/www.scientific.net/DDF.

10. Structure, thermal stability, and state of grain boundaries of copper subjected to highpressure torsion at cryogenic temperatures / A. V. Stolbovsky, V. V. Popov, E. N. Popova, V. P. Pilyugin // Bulletin of the Russian Academy of Sciences: Physics. - 2014. - Vol. 78, iss. 9. P. 908-916. - DOI: 10.3103/S1062873814090299.

11. Saturation of fragmentation during severe plastic deformation / R. Pippan, S. Scheriau, A. Taylor, M. Hafok, A. Hohenwarter, A. Bachmaier // Annual Review of Materials Research. 2010. - Vol. 40. - P. 319-343. - DOI: 10.1146/annurev-matsci-070909-104445.

12. Stolbovsky A. V., Popov V. V., Popova E. N. Structure and Thermal Stability of Tin Bronze Nanostructured by High Pressure Torsion // Diagnostics, Resource and Mechanics of materials and structures. - 2015. - Iss. 5. - P. 118-132. - DOI: 10.17804/2410-9908.2015.5.118-132. URL: http://dream-journal.org/issues/2015-5/2015-5_52.html (accessed 30.10.2017).

13. Evolution of the Structure of $\mathrm{Cu}-1 \% \mathrm{Sn}$ Bronze under High Pressure Torsion and Subsequent Annealing / V. V. Popov, E. N. Popova, A. V. Stolbovsky, R. M. Falahutdinov // Physics of Metals and Metallography. - 2018. - Vol. 119. - P. 358-367. - DOI: 10.1134/S0031918X18040154.

14. Popov V. V., Stolbovsky A. V., Popova E. N. Structure of nickel-copper alloys subjected to high-pressure torsion to saturation stage // Physics of Metals and Metallography. - 2017. Vol. 118. - P. 1073-1080. - DOI: 10.1134/S0031918X17110114.

15. Structure and thermal stability of $\mathrm{Cu}$ after severe plastic deformation / V. V. Popov, A. V. Stolbovsky, E. N. Popova, V. P. Pilyugin // Defect and Diffusion Forum. - 2010. - Vol. 297301. - P. 1312-1321. - DOI: 10.4028/www.scientific.net/DDF.

16. Structure, thermal stability, and state of grain boundaries of copper subjected to highpressure torsion at cryogenic temperatures / A. V. Stolbovsky, V. V. Popov, E. N. Popova, V. P. Pilyugin // Bulletin of the Russian Academy of Sciences: Physics. - 2014. - Vol. 78, iss. 9. P. 908-916. - DOI: 10.3103/S1062873814090299. 
17. Kon'kova T. N., Mironov S. Y., Korznikov A. V. Room-temperature instability of the structure of copper deformed at a cryogenic temperature. Russian metallurgy (Metally). - 2011. Vol. 2011, iss. 7. - P. 689-698. - DOI: 10.1134/S0036029511070081.

18. Structure Evolution and Stability of Copper Deformed at $80 \mathrm{~K} / \mathrm{L}$. M. Voronova, T. I. Chashchukhina, M. V. Degtyarev, V. P. Pilyugin // Russian Metallurgy (Metally). - 2012. Vol. 2012, iss. 4. - P. 303-306. - DOI: 10.1134/S0036029512040131.

19. Deformation and dynamic recrystallization in copper at different deformation rates in Bridgman anvils / T. I. Chashchukhina, L. M. Voronova, M. V. Degtyarev, D. K. Pokryshkina // Physics of Metals and Metallography. - 2011. - Vol. 111, iss. 3. - P. 304-313. - DOI: 10.1134/S0031918X11020049.

20. Stolbovsky A., Farafontova E. Statistical analysis method of the grain structure of nanostructured single phase metal materials processed by high-pressure torsion // Sol. Stat. Phenomena. - 2018. Vol. 284. - P. 425-430. - DOI: https://doi.org/10.4028/www.scientific.net/SSP.284.425.

21. Stolbovsky A., Farafontova E. Statistical analysis of histograms of grain size distribution in nanostructured materials processed by severe plastic deformation // Sol. Stat. Phenomena. 2018. - Vol. 284. - P. 431-435. - DOI: https://doi.org/10.4028/www.scientific.net/SSP.284.431. 